\title{
Geopolymer leaching in water and acetic acid
}

\author{
Neven Ukrainczyk ${ }^{1 *}$, Oliver Vogt ${ }^{1}$ \\ 1 TU Darmstadt, Institute of Construction and Building Materials, 64287 Darmstadt, Germany
}

Received: 30 July 2020 / Accepted: 17 December 2020 / Published online: 21 January 2021

(C) The Author(s) 2021. This article is published with open access and licensed under a Creative Commons Attribution 4.0 International License.

\begin{abstract}
Elemental leaching of metakaolin based geopolymers was investigated by immersing hardened paste specimens in a solution. For this, pure water and 0.1 molar acetic acid solutions were replenished ten times distributed over 56 days in total. Dissolution and diffusion of the elements through and from the geopolymer paste into the surrounding solutions was investigated on cross-sections of specimens by SEM-EDS microscopy, indentation, X-ray powder diffraction analysis and measuring the eluted elements by ICP-MS when replenishing the solution over time. The presented new methodological approach thus combines the dissolution rate kinetics obtained via wet chemistry (ICP) with the complementary solid state characterisation methods to gain new insights into the complex geopolymer dissolution mechanisms.

Results indicated a relatively small leachability of geopolymers, limited only to the surface layer which is directly exposed to the aggressive solution, while the more inner parts of the geopolymer framework remain intact. Elemental maps revealed dissolution of aluminates that occurred across the outermost surface parts of the sections, while potassium leached out gradually but reached deeper inner parts. However, there was still a high portion of potassium being left bonded inside the geopolymer, even for the harsh acidic conditions, limited by the diffusion-reaction mechanism which took place within the geopolymer. The obtained experimental results represent a first approach towards feeding reactive transport numerical modeling approaches still to be developed for simulating leaching and degradation of geopolymer materials when exposed to water or acidic solutions.
\end{abstract}

Keywords: Geopolymers; Leaching; Dissolution rates; Acid attack; Degradation; Microstructure

\section{Introduction}

Geopolymer is a cement free inorganic binder that has a great potential as a special application material with strong resistance against acids [1-3]. Acid resistance is attributed to the stability of employed low-calcium type of aluminosilicate polymers. Geopolymers are inorganic binders composed by a two-component mixture of a reactive solid powder and an alkaline activator solution (Fig. 1). The powder ideally consists mainly of amorphous/reactive aluminosilicates (chemicaly $\mathrm{SiO}_{2}$ and $\mathrm{Al}_{2} \mathrm{O}_{3}$ ). Geopolymer is considered as a binder system, as it can be processed similarly like conventional cement. The low calcium content is a vital feature that distinguishes geopolymers from alkali activated binders. If hardening has to take place at room temperature, and the geopolymers with low to no calcium content are desired, metakaolin is the most used powdery solid precursor [4]. The chemical reaction between the two main components, solid aluminosilicates and alkali-silicate solution, is called geopolymerization. Geopolymerization is fundamentally different from the hydration of cement and can be explained in following simplified steps (Fig.1). First, the $\mathrm{OH}^{-}$ions of the activator solution dissolve the amorphous components of the reactive solid. This breaks the aluminosilicate bonds within the solid and dissolves aluminate and silicate species, e.g. monomers like orthosilicic acid $\left[\mathrm{Si}(\mathrm{OH})_{4}\right]$ and tetrahydroxo-aluminat-ion
$\mathrm{Al}(\mathrm{OH})_{4}^{-}$in a solution, as well as other more complex species such as dimer, trimer, and in various combinations of aluminate and silicate basic building units. In the progress of the successive polycondensation reaction, the dissolved species polymerise and precipitate from the solution to form a geopolymer network exhibiting highly cross-linked polymerisation degrees. Geopolymer binders harden by polycondensation reaction of aluminate and silicate tetrahedrons, cross-linked via oxo-bridging bounds, where a negative charge of aluminate units is compensated by alkali cation precursor (Fig. 1).

Cation exchange of alkalis and solubility of hardened geopolymer play a crucial role in leaching, acid attack and waste stabilisation performance. Leaching of alkalies from geopolymer may reduce alkalinity of pore solution, thus inducing possible corrosion of reinforcing steel in concrete [56]. However, this does not affect the load bearing capacity of the geopolymer matrix alone, if the drop in $\mathrm{pH}$ does not dissolve significant amounts of $\mathrm{Al}$ and $\mathrm{Si}$ from this binder framework. Numerical modeling of the alkali leaching and ion-exchange reactive-diffusion process [7] showed that there is high amount of bonded alkalies that might maintain the $\mathrm{pH}$ inside the geopolymer, which is important for reinforcement steel corrosion.

\footnotetext{
* Corresponding author: Neven Ukrainczyk, Email: ukrainczyk@wib.tu-darmstadt.de
} 


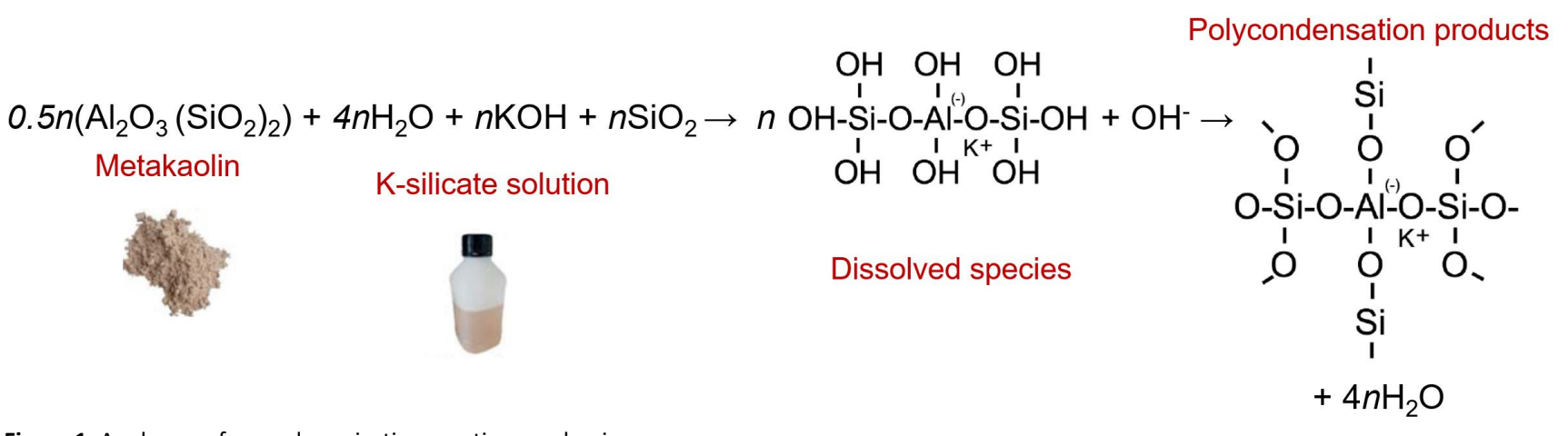

Figure 1. A scheme of geopolymerisation reaction mechanism.

Geopolymer binders are particularly interesting as an innovative alternative to cementitious materials used in applications which are frequently exposed to various acids, e.g. organic [8,9] or mineral acids [10-15]. Acetic acid attack is a typical rapid acidic mechanism resulting in a highly porous degraded surface layer due to leaching of the highly soluble acetate salts, it's complexation and $\mathrm{pH}$ buffering characteristics. Koening et al. (2017 [9]) studied comparatively the degradation of sodium $(\mathrm{Na})$-silicate activated binders based on various mixtures of slag (as high $\mathrm{CaO}$ component) and fly ash (as low $\mathrm{CaO}$ component) attacked by organic acids. The chosen representative typical exposure conditions in agricultural constructions were simulated by a mixture of acetic, propionic and lactic acid having a $\mathrm{pH}$ of 3 . They found that the resistance to the organic acids increases with decreasing $\mathrm{CaO}$ content of the binder. They also highlighted an improvement in mechanical properties of the near surface transition zone with acid exposure time attributed to acid induced phase transformations. Although significant research efforts have already been done so far on geopolymers [1,2,7-17], there is a lack of research evaluating the solubility of geopolymer binders. Therefore, this paper aims to better understand the chemical dissolution processes happening in the geopolymers, when exposed to pure water and acetic acid environments. Microstructural alterations were analysed on specimens after the leaching exposure, by analysing the cross-section degradation depth profiles using elemental mappings with energy dispersive spectroscopy in scanning electron microscopy (SEM-EDS), nano- and micro-indentation and $\mathrm{X}$-ray powder diffraction analysis. The concentration of eluted ( $\mathrm{Al}$, Si and K) elements in exposure solution at various times of replenishing was measured by ICP-MS.

\section{Methodology}

\subsection{Materials}

Chemical composition of the geopolymer raw materials is given in Table 1. Metakaolin powder was pressed in tablets and evaluated by X-ray fluorescence analysis (XRF) using a wavelength dispersive X-ray fluorescence spectrometer (PANalytical 2404). Metakaolin had a specific surface of 26000 $\mathrm{cm}^{2} / \mathrm{g}$ and loss on ignition of $1.9 \%$. As alkaline activator, potassium silicate solution was used with solid content of 45 $\%$, molar $\mathrm{SiO}_{2} / \mathrm{K}_{2} \mathrm{O}$ ratio of 1.5 , viscosity of $20 \mathrm{mPas}$ and a density of $1.51 \mathrm{~g} / \mathrm{cm}^{3}$. Ultra-pure deionized water, with resistivity of greater than $18.1 \mathrm{M} \mathrm{Ohm} \mathrm{cm}$ was used for leaching tests.

Table 1. Chemical composition of Metakaolin and K-based silicate solution (waterglass).

\begin{tabular}{|l|l|l|}
\hline Material & Metakaolin & K- waterglass \\
\hline $\mathrm{SiO}_{2}$ & 50.4 & 22 \\
\hline $\mathrm{Al}_{2} \mathrm{O}_{3}$ & 40.5 & \\
\hline $\mathrm{CaO}$ & 0.1 & \\
\hline $\mathrm{TiO}_{2}$ & 1.5 & \\
\hline $\mathrm{Fe}_{2} \mathrm{O}_{3}$ & 2 & \\
\hline $\mathrm{MgO}$ & 0.1 & \\
\hline $\mathrm{Na}_{2} \mathrm{O}$ & 0.1 & \\
\hline $\mathrm{K}_{2} \mathrm{O}$ & 0.1 & 23 \\
\hline $\mathrm{H}_{2} \mathrm{O}$ & - & 55 \\
\hline
\end{tabular}

\subsection{Geopolymer specimen preparation}

Geopolymer pastes were mixed in waterglass to metakaolin mass ratio of one, poured in plastic molds, cylinders $8.5 \mathrm{~mm}$ in diameter and $3 \mathrm{~cm}$ in height, and cured at room temperature for 28 days. Cured geopolymers cylindrical specimens were embedded in epoxy resin. After the resin was cured, each specimen was sectioned to final dimensions of 2 $\mathrm{cm}$ in height ( $8.5 \mathrm{~mm}$ in diameter) using a low speed diamond saw to expose one base of the cylinder to leaching solution (Fig. 2).

Care was taken to limit carbonation effects not only during specimen's preparation, but more importantly during the leaching experiments and sample storage. This was done by using nitrogen air flow whenever needed, e.g. when opening the curing or leaching containers, especially during sampling of the replenishment solutions (described in next section). The solid samples were stored in sealed containers that were purged with nitrogen flow and the gas environment was regulated by concentrated $\mathrm{KOH}$ solution to bind any $\mathrm{CO}_{2}$.

\subsection{Leaching experiments}

Diffusion and dissolution of the elements from the geopolymer paste was investigated by immersing geopolymer cylindrical specimens (diameter $8.5 \mathrm{~mm}$, height 2 $\mathrm{cm}$ ) in ultra-pure water and 0.1 molar acetic acid (measured $\mathrm{pH}$ of 2.8) by replenishing the solution with eluted elements over time. 


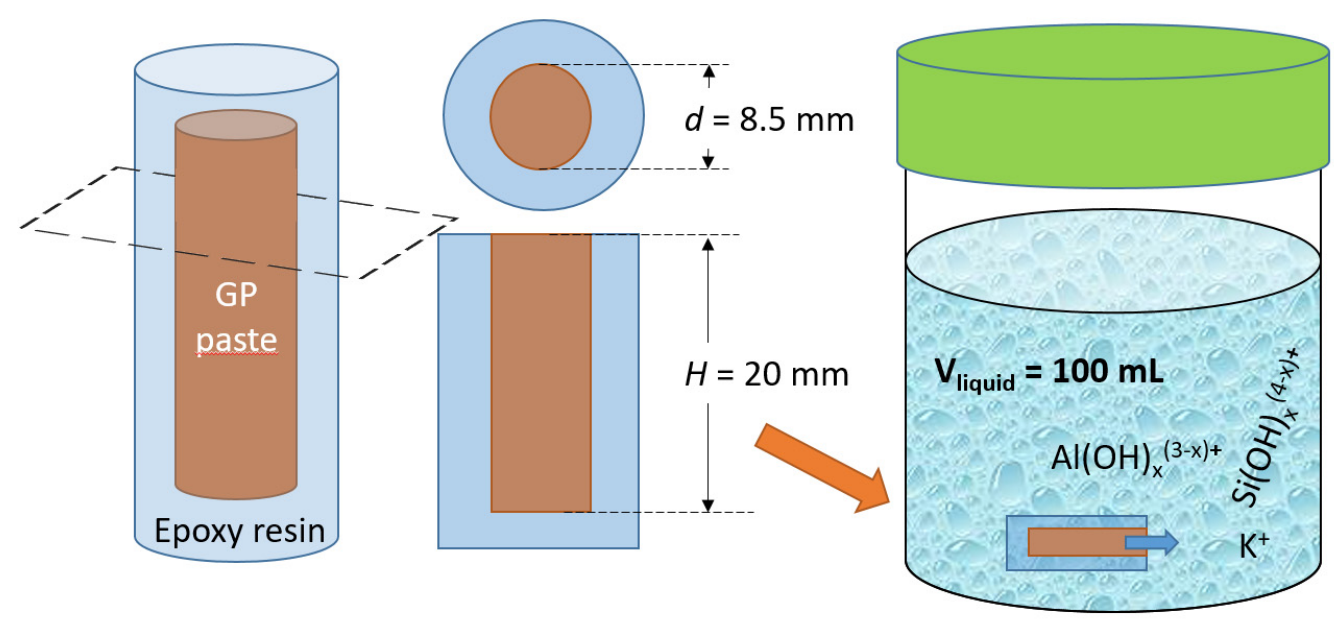

Figure 2. A scheme of the one-dimensional diffusion leaching test: cylindrical geopolymer specimens are immersed in $100 \mathrm{~mL}$ solution for up to 56 days of exposure, where the solution was replaced with a fresh one in ten time intervals.

One-dimensional diffusion conditions were obtained by exposing the one base of the cylindrical geopolymer specimens, while the other surfaces of the cylinder are covered by epoxy resin. Each sample was immersed in $100 \mathrm{~mL}$ of 0.1 molar acetic acid maintained at $21^{\circ} \mathrm{C}$. After $1-56$ days of exposure, in 10 time intervals $(1,3,7,14,21,28,35,42,49$, 56 days), the acetic acid solution was replaced with a fresh one.

As there is no standard/recommendation methodology to test the leaching of geopolymers the time intervals were chosen according to semi-dynamic leaching method for Solidified Low-level Radioactive Waste (e.g. ANSI, Measurement of the Leachability of Solidified Low-level Radioactive Waste by a Short-term Test Procedure, ANSIANS-16.1, 2003). The acidic solution was replenished to achieve quick results by accelerating the deterioration process, and to sample the dissolved elements. For the same reason, the initial high acid concentration chosen (100 mM), which may not be representative to the real-field application was adopted for this study, and to account for the neutralisation reaction between geopolymer and the acid. The closed cylindrical cups which contained the specimens immersed in the solution were closed in polyethylene cylindrical cups (Figure 2 right, having a diameter of $5 \mathrm{~cm}$ ) which were slowly rotated at a rotation speed of $0.5 \mathrm{~Hz}$ by means of a chemical laboratory rotation device. The rotation was applied continuously during the whole duration of the leaching experiment, disrupted only for measurements and solution replacement at the specified time intervals.

After reaching the planed leaching intervals, two separate eluate solution samples were taken: 1) for the direct $\mathrm{pH}$ measurement, and 2) eluates for ICP-MS measurement. The $\mathrm{pH}$ of sampled solutions was measured immediately using a pH electrode (Hanna pH 211, United States). The eluates were filtered through a $0.45 \mu \mathrm{m}$ cellulose acetate membrane and diluted for ICP-MS analysis. The filtered liquid samples were analyzed via inductively coupled plasma - mass spectrometry
(ICP-MS; measurement uncertainty was $<1 \%$ for an order of magnitude more concentrated elauats leached in acetic acid than in pure water, where it was $<5 \%$ ) done in an accredited laboratory (according to DIN EN ISO/IEC 17025) using standard method following EN ISO 17294-2: 2005-02. The quantification detection limits were 10,10 , and $50 \mathrm{\mu g} \mathrm{L}^{-1}$ for $\mathrm{Al}, \mathrm{Si}$, and $\mathrm{K}$, respectively.

\subsection{SEM-EDS elemental mappings, nano- and micro-indentation}

SEM-EDS were performed on cross-sections of aged, i.e. leached specimens. The cut cross-sections were impregnated with low viscosity (two-component) liquid epoxy using a vacuum impregnation device. To reveal the sample surface, the hardened epoxy polymer was first polished at a rotational speed of $300 \mathrm{rpm}$ using a resin bonded diamond disc (hardness range HV 150 to 2000) mounted on the semiautomatic grinding-polishing machine (Struers). Then, it was polished consecutively on a lubricated cloth mounted on a polishing machine to the desired level using an automated polycrystalline diamond spray of $9 \mu \mathrm{m}, 3 \mu \mathrm{m}$, and $1 \mu \mathrm{m}$ size at a rotational speed of $150 \mathrm{rpm}$. In the case of SEM (Zeiss EVO LS25), the polished section of the deteriorated paste specimens were prepared to collect EDS elemental mapping images (EDAX, AMETEK, Berwyn, USA) and back-scattered electron (BSE) images.

Operating conditions in the environmental SEM were kept at low vacuum mode at $10 \mathrm{~Pa}$ to prevent charging effects on the samples, while eliminating the use of conductive coatings. Elemental mappings were performed under $1.1 \mathrm{nA}$ (probe spot current) and $13 \mathrm{kV}$ using a dwell time of $500 \mu \mathrm{s}$ for each pixel, with $512 \times 400$ pixels in a frame, repeated for 240 frames.

A ZAF correction was applied to minimize the interaction effects between sample surface and electron beam, using an algorithm that accounts for the atomic number $(Z)$, the absorption correction $(A)$ and the fluorescence correction $(F)$. 
These corrections are used for matrix effects such as differences in mean atomic number, in absorption of $x$-rays and production of $\mathrm{x}$-rays or $\mathrm{x}$-ray fluorescence. Apart from internal standards, also an external reference standard calibration was employed.

The microstructure of the reference and acid attacked samples was examined mechanically by following two methods: 1) Nano Indenter (Agilent G200) with a diamond Berkovich tip and 2) by a Vickers Micro indenter (Fischerscope Hardness tester $\mathrm{H} 100$ ). Both nano and micro indentation tests were performed on an epoxy impregnated and polished samples. Operating conditions for Vickers geometry included about 20 indents with $1 \mathrm{~N}$ loading. In the Berkovich nanoindentation testing about 50 indents were conducted according to Muthu et al. [18], using the center-to-center spacing between the indents at $50 \mu \mathrm{m}$. The maximum load of $45 \mathrm{mN}$ was held for $10 \mathrm{~s}$, followed by $10 \mathrm{~s}$ of unloading period, leading to a penetration depth range of $500-1000 \mathrm{~nm}$ into the sample depending on the hardness. The elastic modulus was calculated using the model parameters from Muthu et al. [18]. The hardness is calculated as a ratio of employed force over the indented surface area.

\subsection{X-ray powder diffraction of sliced cross sections}

Powder samples were sectioned from geopolymers specimens using high precision low-speed saw (Buehler Isomet-1000) in depth sections of 0-1 mm, 1-2 mm and 2-3 $\mathrm{mm}$. The sections were ground to a fine powder $(<50 \mu \mathrm{m})$. Powder X-ray diffraction (XRD) was performed on Bruker D2 Phaser (Hamburg, Germany), configured with CuKa $\alpha_{1,2}$ radiation (40 kV and $10 \mathrm{~mA}$ ), linear Lynxeye detector (5 degrees opening). All samples were measured with 0.02 two theta step size and measurement time of $2 \mathrm{~s} / \mathrm{step}$.

\section{Results and discussion}

\subsection{Leaching rates}

Results of measuring the concentration of eluted elements in the replenishing solutions over time, are presented in Figure $3 a$ and $3 b$ for the acid case and the pure water case, respectively. It can be observed that the different curves have similar trend, but are shifted in concentrations, where the exposure to acetic acid results in an order of magnitude higher concentrations than for the pure water case. In both cases, the concentration at 1st day is higher than the one obtained after 3 days, and after it increases again reaching maximum for 14 days, monotonically decaying subsequently. The evolution of $\mathrm{pH}$ exhibits similar trend to the one for the potassium concentrations.

Potassium leaching rates are calculated from the results in Figure 3 by considering the concentration expressed in $\mathrm{mg}$ per $100 \mathrm{~mL}$ of employed leaching volume (i.e. the results in Figure 3 are divided by 10), and dividing this leached mass with the time interval and specimen's leaching surface area (56.7 $\mathrm{mm}^{2}$ ). The leaching rates results in Figure 4 show monotonical decrease with time, indicating that the non-monotonical trend observed in Figure 3 is due to the different time intervals used in leaching test, which is initially shorter, namely 1, 2 and 4 days, followed by repeating a fixed interval of 7 days. The logarithmic plot indicates a fair exponential behavior. Only the initial point (1 day) deviates significantly from this behavior having a much higher values. This could be explained by an initial leaching of $\mathrm{K}$ which is excessive in the pore solution, and later with leaching time the binded $\mathrm{K}$ is being leached by cation exchange and geopolymer network dissolution mechanism [6-8,14-17]. The comparison of the leaching rates in 0.1 molar acetic acid and pure water clearly shows an order of magnitude difference. To the best of our knowledge these rates are not readily being available in literature, and especially not from K-based metakaolin based geopolymer paste specimens (in 1 dimensional experimental set-up), complemented by changes in geopolymer nearsurface layers obtained by cross section elemental mapping analysis (in next section 3.2). The approach proposed here should be used for comparison of the leaching behavior of the wide varieties of the geopolymer designs.

The total amount of (cumulatively) leached $\mathrm{Si}, \mathrm{Al}$ and $\mathrm{K}$ elements was calculated as a sum of measured concentrations in replenished solutions (by ICP) and related to $100 \mathrm{~mL}$ of the leaching solution, i.e. per specimen. The results for the acid exposure are: $31.75 \mathrm{mg} \mathrm{K} / 100 \mathrm{~mL}, 7.48$ $\mathrm{mg} \mathrm{Al} / 100 \mathrm{~mL}$, and $9.57 \mathrm{mg} \mathrm{Si} / 100 \mathrm{~mL}$. The case of leaching in pure water resulted in the significantly (an order of magnitude) lower total amounts of leached elements: 2.83 $\mathrm{mg} \mathrm{K} / 100 \mathrm{~mL}, 0.305 \mathrm{mg} \mathrm{Al} / 100 \mathrm{~mL}$, and $0.262 \mathrm{mg} \mathrm{Si} \mathrm{/} 100$ $\mathrm{mL}$. Also the $\mathrm{K}$ leaching rates (Figure 4 ) are an order of magnitude lower in water than in acid.

The cumulative results show that potassium is leached about 4 and 10 times higher than silicium and aluminium, in acid and water, respectively. In acid case the aluminium had somewhat (22\%) lower cumulative concentrations than silicium. This turned out to have an opposite trend when leaching in pure water, where the aluminium reached (16\%) higher cumulative concentrations than silicium. This indicates to the different behaviour of aluminium and silicium solubilities from the geopolymer gels as a function of $\mathrm{pH}$, resulting in increase of the $\mathrm{Al} / \mathrm{Si}$ ratio of the geopolymer solids when leached in the alkaline conditions, while this ratio decreases upon leaching in acidic conditions.

The persistence in bonding of potassium (exponential leaching rates in Figure 4) and low leachability of Si and Al from geopolymer shows that the degradation process is limited by a diffusion-reaction mechanism which takes place throughout the geopolymer (as shown next by SEM-EDS elemental maping results). Even for the very aggressive environment studied, i.e. 0.1 molar acetic acid, $\mathrm{K}$ is still bounded in inner parts of geopolymer specimen, resulting in a monotonical exponential decay of the leaching rates with time. The final leaching rates measured after 10 solution replacements are almost two order of magnitudes lower than the initial leaching rates, both in water and acid exposure conditions. 

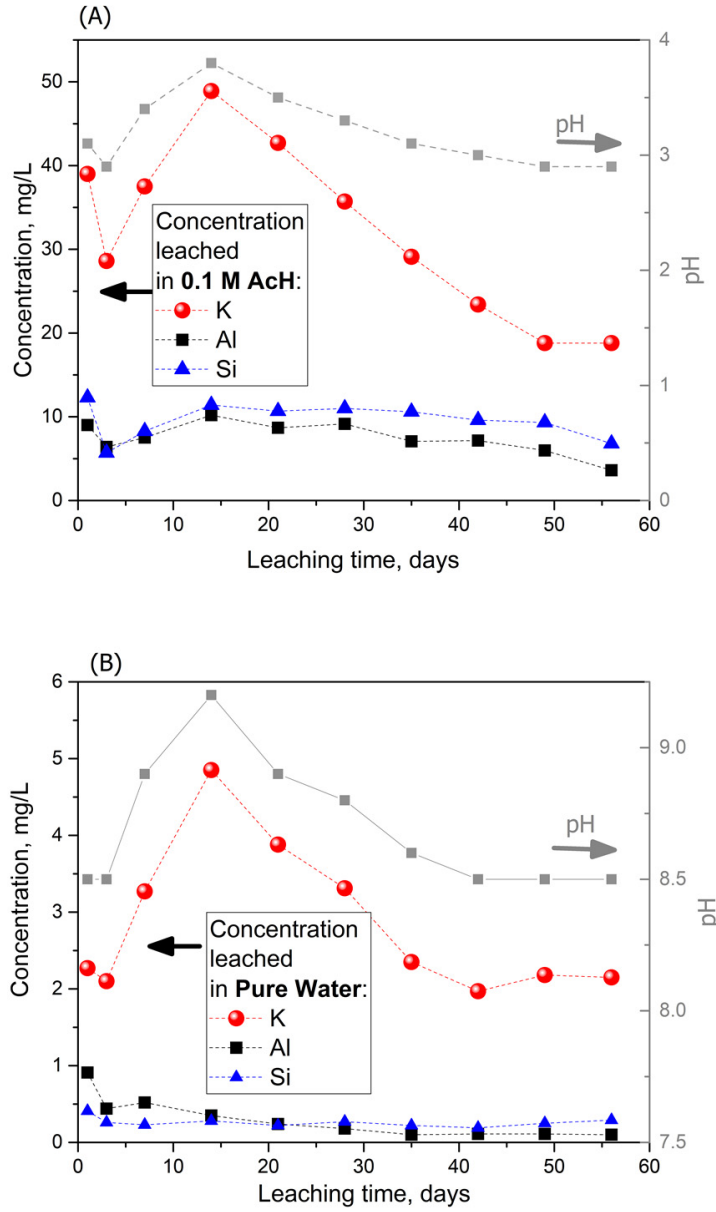

Figure 3. Concentration of eluted elements and the $\mathrm{pH}$ of the replenishing solutions over time. A) Leaching in acetic acid $(\mathrm{AcH})$ and B) in pure water.

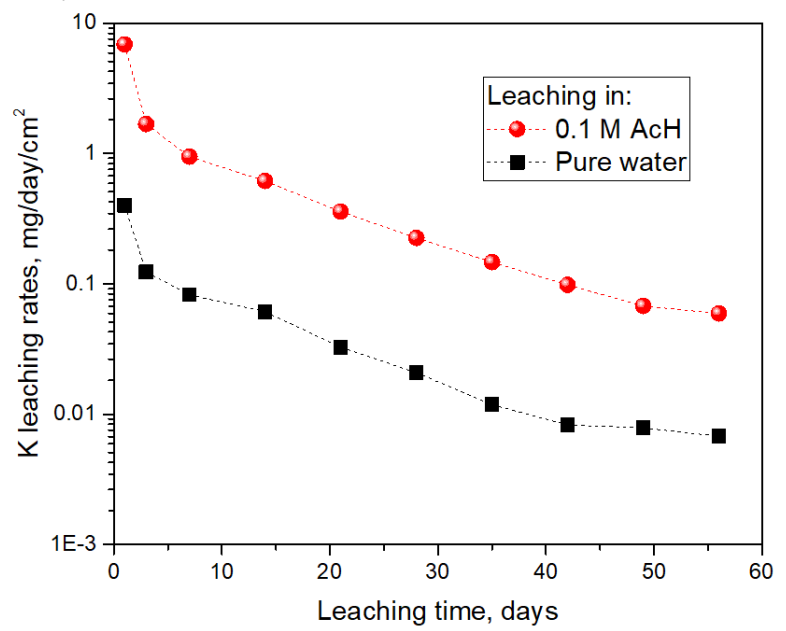

Figure 4. Potassium leaching rates calculated from results in Figure 3, by normalizing the concentration (expressed in $\mathrm{mg}$ per $100 \mathrm{~mL}$ of employed leaching volume) per time interval and specimen's leaching surface area.

\subsection{SEM-EDS elemental maps}

High-resolution EDS elemental maps and BSE images on cross-sections revealed the deterioration degree and phase changes in the leached geopolymer hardened pastes after 56 days of exposure to 0.1 molar acetic acid (Figs. 5 - 8 ) and pure water (Figs. 6 and 9). The outermost surface exposed to the external solution is represented by the left edge of the SEMEDS image in Fig. 7 for the acid attack and Figs. 9 for the leaching in pure water. Thus, the acid/water penetrates always from the left side of SEM-EDS images, and the images are taken at different degradation depths (only on figure 6 the solution-geopolymer interface is orientated on the top side). The stacking of mappings for obtaining the profile elemental analysis is depicted in Figure 7. For clarity, this example shows only a stack of first three SEM-EDS mapping images (out of 4) of the specimen's cross sections. The left boundary of the image represents the specimen's surface boundary section exposed to leaching in acetic acid. For example, in the first left image, the degradation depth is $2.3 \mathrm{~mm}$, meaning that the left edge of the image has $0 \mathrm{~mm}$, and the right edge has $2.3 \mathrm{~mm}$. The height of images is typically $3 \mathrm{~mm}$. Next image in Fig. 7 goes further in the depth with small overlap.

Comparison of Figures 7 and 9 shows clearly that the deteriorated layer under acid attack was significantly more affected than under pure water exposure, as expected. The leftmost parts, near the specimen's surface exposed to the external solution, and especially under acid attack, appeared to be highly porous. Geopolymer under water, meanwhile, was less degraded. The remaining main elements in the deteriorated layer of the specimens were clearly revealed by their elemental maps (Figs. 7-9). The major elements that were most affected by the leaching were $\mathrm{Si}$, Al, and K. Some increased localised Na concentrations (orange color in Figs 7 and 9) could be observed originating from clay impurities present in the metakaolin raw mineral. However as they total amounts are very small (Table 1) Na was not considered in further analyses. The carbon (C) presence indicates the epoxy, which corresponds to increased porosity. On the other hand, the major elements that leach out due to acid or water exposure were also clearly noticed in the EDS mapping results. On the whole, the elemental maps confirmed the gradual leaching of potassium, followed by dealumination as well as (re-) precipitation of Si- or Al-rich geopolymer gel in the near-surface deteriorated layer, for the acid or water case, respectively (shown later when discussing the results of the statistical elemental depth profile distribution). 

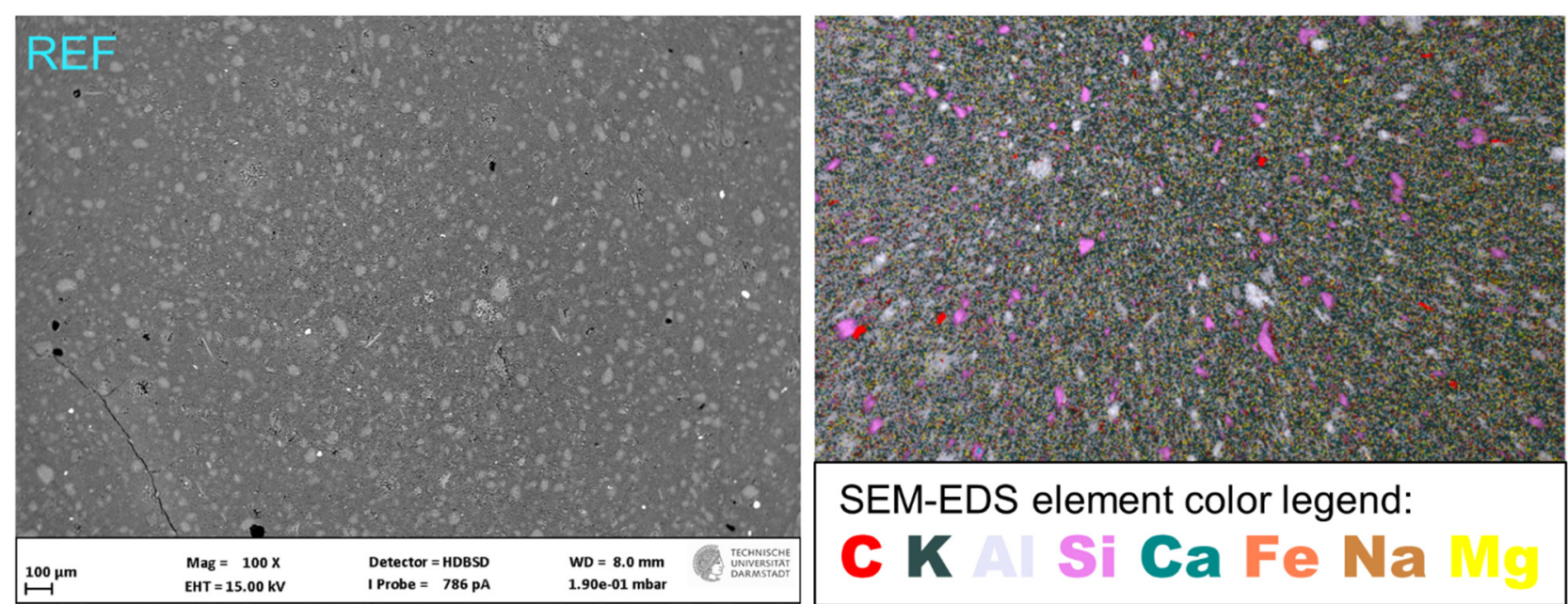

Figure 5. SEM-BSE (left) and elemental mapping (right) for the reference geopolymer.
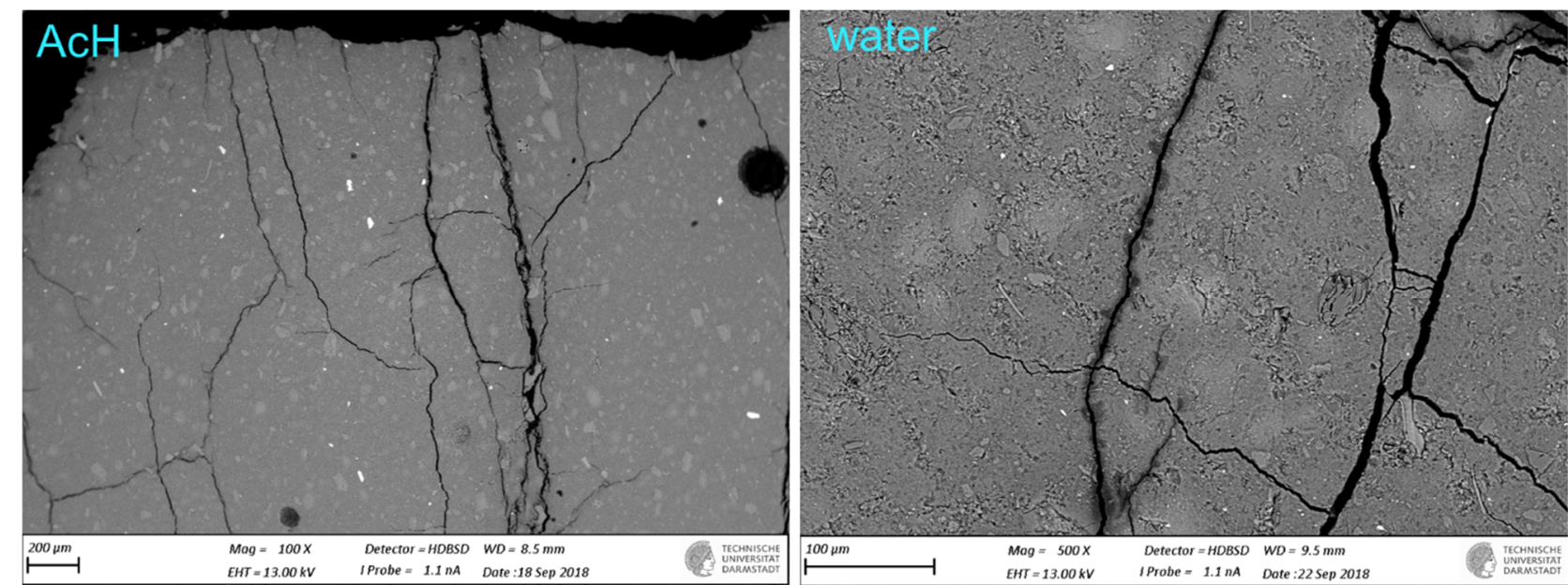

Figure 6. SEM-BSE images of the specimen's surface layers exposed to leaching in acetic acid (AcH, left) and Water (right). The acid penetrates from the upper side of the image.

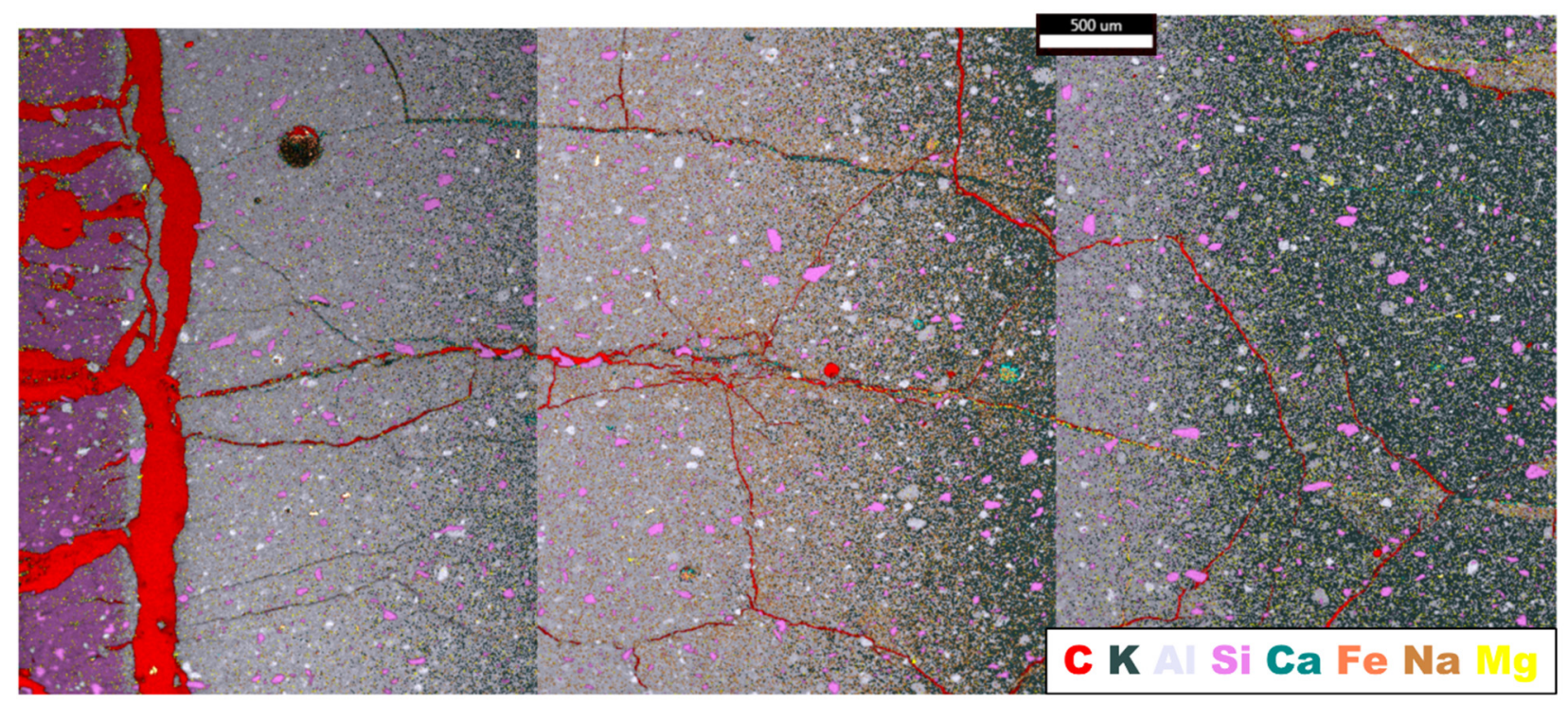

Figure 7. A stack of three SEM-EDS mapping images of the specimen's cross sections for the analysis of elemental depth profiles. The left boundary of the image represents the specimen's surface boundary section exposed to leaching in acetic acid. The element color legend is shown in rightbottom corner of the image. 


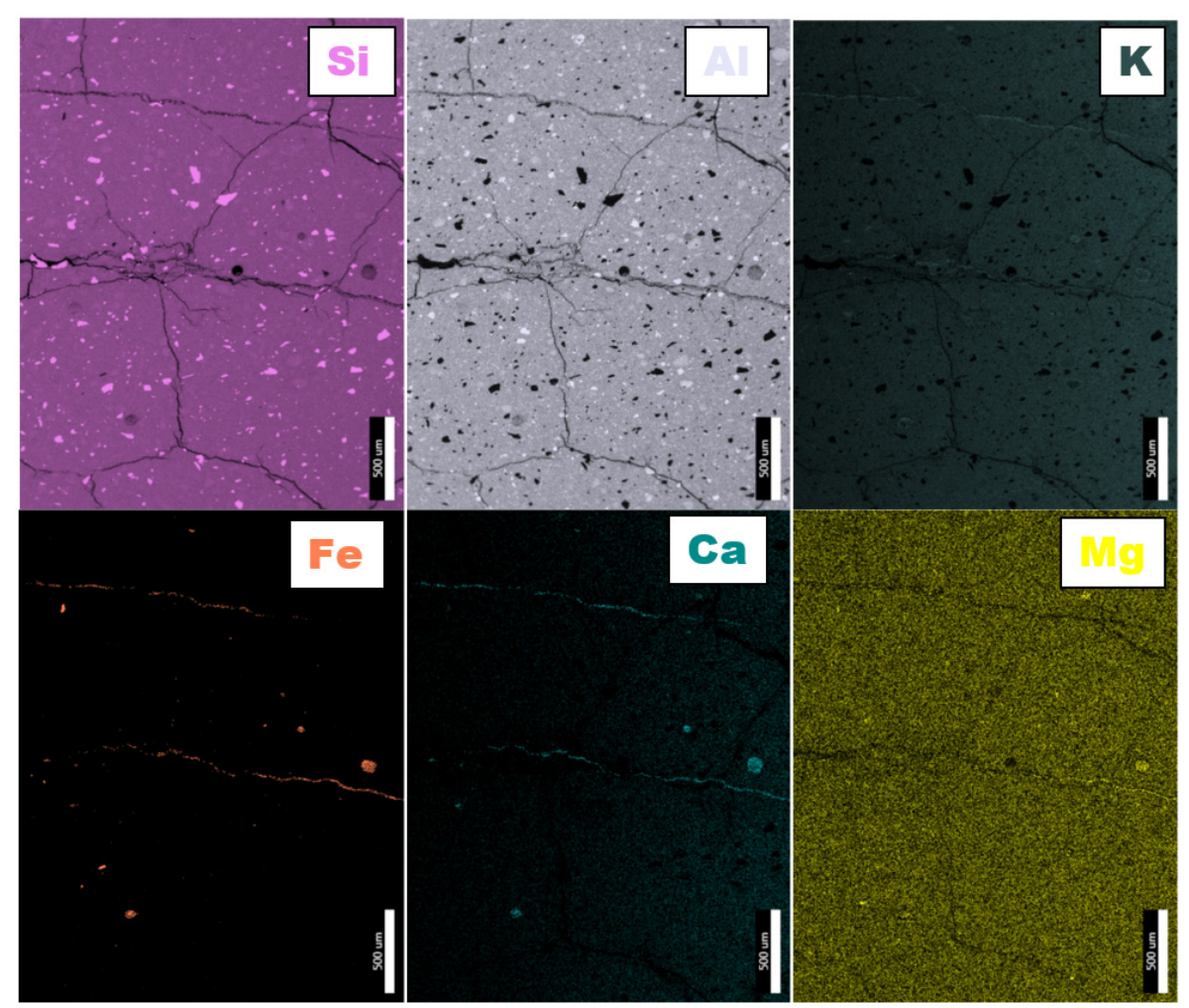

Figure 8. Separation of individual elemental maps taken from the middle (stack) image of the Fig 7.

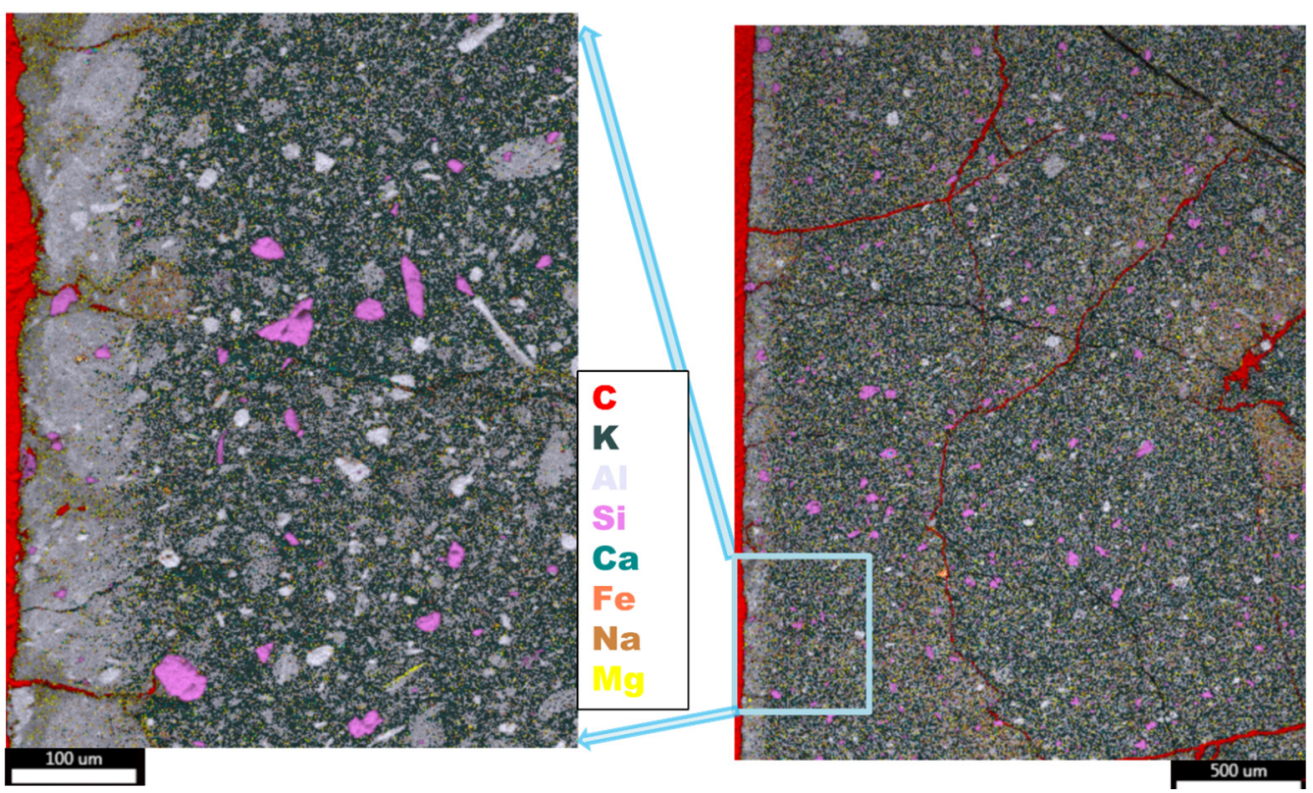

Figure 9. SEM-EDS elemental mapping of a cross section after the exposure to pure water. The geopolymer-water interface is on the left side of the image.

The SEM images in Figures 5-9 evidently revealed the occurrence of lengthy cracks that are connecting the top surface layer of the sound inner zone. These cracks could be attributed to the shrinkage resulting from the dissolution of geopolymer elements, as well as due to drying shrinkage conditions used for sample preparations. Comparison of the Figures 5-7 and 9 shows that the reference sample (Figure 5) had by far lower tendency to crack formation than the samples leached in water (Figure 9), followed by acid exposure conditions (Figure 7). In acid case substantial detachment was observed on the top surface layer of the damaged specimen. The dissolution induced shrinkage caused by the acetic acid attack resulted in differential stresses, putting the geopolymer paste specimen under tension. Shorter and thinner cracks formed when the tensile stress is enough to induce fracture. Figure 7 and 8 demonstrate that the lengthy cracks have a crucial role in forming preferential diffusion paths. This is more clearly 
observed in Figure 8 by means of separation of individual elemental maps, which correspond to the middle (stack) image extracted from the combined Fig 7. The individual maps clearly show the roundish particles which are rich in CaFe-K-Mg and poor in Si-Al. They can be attributed to the unreacted impurities present in the raw clay powder material. Two lengthy (horizontal) cracks can be observed which are filled with Ca-Fe-K-Mg dissolved species which are diffusing towards upper surface layers. The zoom in on the surface layer in Figure 9 shows the random distribution of unreacted quartz grains (in pink color) embedded in the geopolymer framework, which originate from impurities present in the raw clay powder material (in agreement with X-ray diffraction results). The quartz grains show no signs of dissolution/alteration in the various degradation layers.

Depth profiles of elemental composition (Figs. 10-11) were obtained from statistical analysis of EDS mappings. Micro areas, i.e. micro-regions of interests are defined by depth increments of mostly $300 \mu \mathrm{m}$, and wideness (broadness) of the image, i.e. the image was divided into eight or more smaller regions having smaller depths but same wideness of the original image. To capture the rapid changes at the uppermost degradation areas, the incremental depths of those regions of interests were made smaller than $300 \mu \mathrm{m}$ (down to $125 \mu \mathrm{m}$ and $60 \mu \mathrm{m}$ for the acid and the water case, respectively). However, it must be stressed that in order to have a good statistics of the image, a representative surface area should be large enough to homogenize (average) the microscopic heterogeneities. The analysis area was kept at $300 \times 3000 \mu \mathrm{m}^{2}$. For each section a mean and corresponding absolute error (i.e. mean \pm one standard deviation) was calculated. The solid horizontal lines in Figures 10 and 11 represent the reference elemental concentration (e.g. K_ref) in the reference sample, i.e. prior to the exposure to acetic acid or pure water [16].

The mass percentage was expressed per $100 \mathrm{~g}$ of binder paste, where the binder paste included carbon (C) as an approximation for porosity. This was related to the epoxy impregnation, wherein the sum of the major (heavier) elements (i.e., $\mathrm{Ca}, \mathrm{Si}, \mathrm{Al}, \mathrm{O}, \mathrm{Fe}, \mathrm{K}, \mathrm{Na}$ and $\mathrm{C}$ ) could roughly approximate a water saturated binder paste, where $\mathrm{C}^{12}$ could be approximated for $\mathrm{O}^{16}$, and $\mathrm{H}^{1}$ was disregarded. The results are considered as semi-quantitative (not absolute), so the changes in concentrations could be well followed.

Degradation profile after leaching in the acetic acid (Fig. 11) shows dissolution of the Al until reaching a depth of about 1 $\mathrm{mm}$, which can be considered as a degradation zone. This zone exhibits sharp de-alumination, namely resulting in the decrease of Al content from $10.7 \%$ to $3.8 \%$, accompanied with simultaneous strong depletion of potassium (K). The concentration profile of potassium indicates a gradual leaching/dissolution of this element, due to combined ion exchange and geopolymer network dissolution [6,8,14-17], all the way till the penetration depth of $9 \mathrm{~mm}$. This value is still slightly below the reference (non degradated) value, as well as measured along the more inner (5-9 mm) depths.

The potassium profile exhibits two main gradients, a higher concentration gradient near the exposure surface, observed until the depth of $5.5 \mathrm{~mm}$, followed by a lower gradient. Correspondingly, the zone 1-5.5 $\mathrm{mm}$ is considered here as a transition zone, where the properties of the geopolymer are not impaired, as the chemical changes are principally due to $K$ leaching. However, in this transition zone it is very interesting to observe also slightly higher concentrations of silicium and aluminium, compared to the far inner zone and the reference sample. However, this could be attributed to dissolution and re-crystallization of Si-richer (Al-richer) aluminosilicate framework, but could be also linked to the dilution effect due to $\mathrm{K}$ leaching. Dissolution and re-crystallization of Si-rich aluminosilicate framework at low pH, i.e. most outer (near surface) degradation area, could not be clearly observed, probably hampered by high heterogeneity of the sample (high scatter of the Si values). Nonetheless, the highest $\mathrm{Si}$ concentration was observed at depth range of $0.25-0.38 \mathrm{~mm}$, indicating to a local re-crystallization of Si-rich aluminosilicate framework, which dissolves again at lower $\mathrm{pHs}$.

Although significant research efforts have already been done so far on GPs [1,2], there is a lack of research evaluating the solubility of geopolymer binders. Research so far covered only empirical GP tests on acid resistance [8-15], thus neglecting the fundamental chemical aspects behind. In this limited context, solubility of GP depends on the $\mathrm{pH}$, for increasing acidic conditions described as follows $[6,8,14]$ :

1. ion exchange reaction between the penetrating acid protons $\left(\mathrm{H}^{+}\right)$and the charge compensating cations $\left(\mathrm{Na}^{+}\right.$ or $\mathrm{K}^{+}$) of the geopolymer framework, along with

2. partial dissolution of the aluminosilicate framework,

3. precipitation of acid anion salts (here in acetic case are highly soluble)

4. crystallization of zeolites causing a decrease in material strength (not observed),

5. dissolution and re-crystallization of Si-rich aluminosilicate framework.

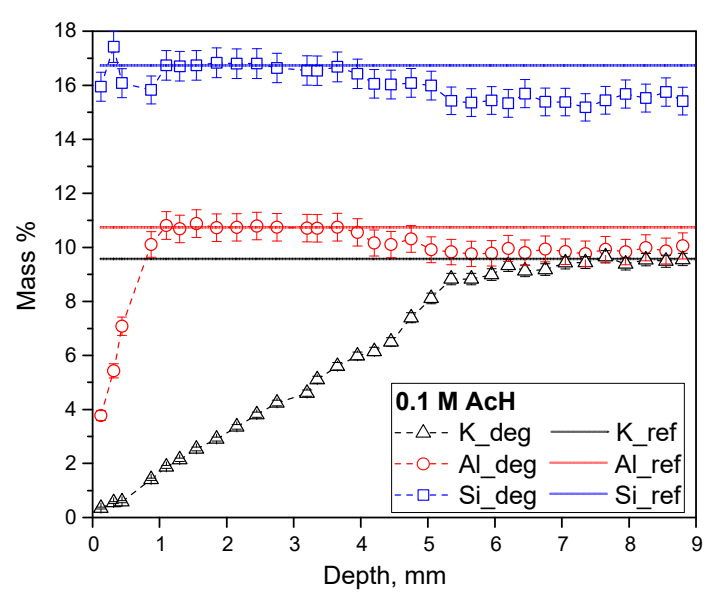

Figure 10. Degradation profile after leaching in the acetic acid $(\mathrm{AcH})$ : semi-quantitative chemical composition as a function of degradation depth, obtained as a statistical analysis of EDS mappings (Fig. 7) taken per various depth ranges (indicated on $x$-axis). 


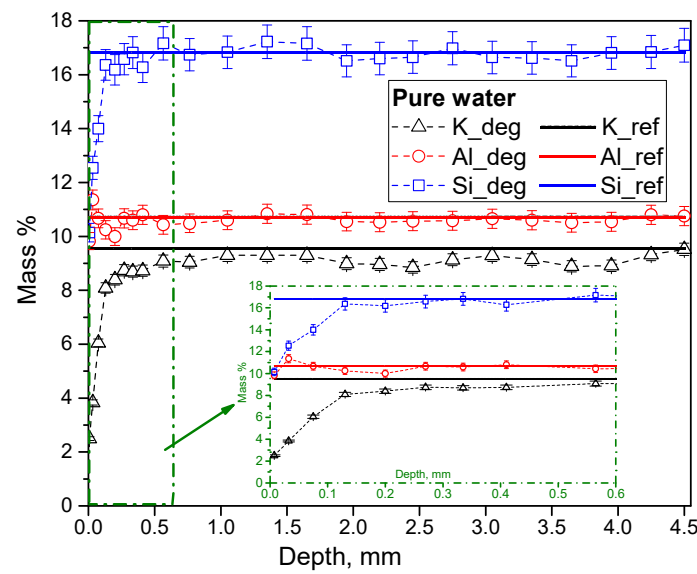

Figure 11. Degradation profile after leaching in ultra-pure water: obtained as a statistical analysis of EDS mappings (Fig. 9) per depth ranges (indicated on $x$-axis).

Degradation profile after leaching in pure water (Fig. 11) shows almost negligible dissolution of $\mathrm{Al}$, but significant dissolution of Si reaching a depth of about $0.16 \mathrm{~mm}$, which is considered here as a degradation zone. This zone exhibits decrease of $\mathrm{Si}$ concentration from $16.8 \%$ to $10.1 \%$, accompanied with simultaneous depletion of $\mathrm{K}^{+}$, from $9.55 \%$ to $2.5 \%$. The concentration profile of potassium $(\mathrm{K})$ indicates a leaching/dissolution, reaching the same penetration depth of $0.16 \mathrm{~mm}$ as observed for Si concentration profile (Fig. 11). We may argue that $\mathrm{K}$ leaching is mostly due to the cation exchange (eq. 2, by aluminate cation species) as the geopolymer dissolution/reorganization (localized at the top surface) involves increased $\mathrm{Al} / \mathrm{Si}$ due to Si dissolution. The ICP results indicated that the total amount of leached $\mathrm{Al}$ was slightly higher than the $\mathrm{Si}$, which can be explained by the localized dissolution of $\mathrm{Si}$, which is limited to the $120 \mu \mathrm{m}$ thin surface layer (Figure 11), and by the leaching out of the Al species diffusing from the further inner parts of the geopolymer.

Elemental profile results demonstrate that the dissolution of Si from the geopolymer gel is much higher in water (alkaline conditions) than in acidic conditions. This led us to propose an extended mechanism for geopolymer dissolution as summarised by equations 1 and 2 (and a GP structure in Figure 1).

$[\mathrm{Si}-\mathrm{O}-\mathrm{Al}-\mathrm{O} . . .]^{-}-\mathrm{K}\left(\mathrm{H}_{2} \mathrm{O}\right)_{n}{ }^{+}+\mathrm{H}_{3} \mathrm{O}^{+} \leftrightarrow[\mathrm{Si}-\mathrm{O}-\mathrm{Si} . .]+.\mathrm{Al}(\mathrm{OH})_{4}{ }^{-}+\mathrm{K}\left(\mathrm{H}_{2} \mathrm{O}\right)_{n}{ }^{+}(1)$

[Si-O-Al-O-Si...] $-\mathrm{K}\left(\mathrm{H}_{2} \mathrm{O}\right)_{n}{ }^{+}+\mathrm{X}^{+}+4 \mathrm{OH}^{-} \leftrightarrow[\mathrm{Si}-\mathrm{O}-\mathrm{Al} . . .]^{-} \mathrm{X}^{+}+\mathrm{Si}(\mathrm{OH})_{4(\text { aq. })}{ }^{+}$ $\mathrm{K}\left(\mathrm{H}_{2} \mathrm{O}\right)_{n}^{+}$

The cation exchanged $\mathrm{X}^{+}$species could most likely be [6] $\mathrm{Al}(\mathrm{OH})_{2}{ }^{+}$or $\mathrm{Al}_{y}(\mathrm{OH})_{z}{ }^{(3 y+z)+}$, but also other minor metal cations present in metakaolin raw materials, such as $\mathrm{Fe}, \mathrm{Ca}, \mathrm{Na}$. Moreover, another mechanism found in literature [1,2] is associated to zeolites crystallization, which causes a decrease in material strength. This result was not corroborated in this study, as it is more commonly found in Na-based GPs $[1,2,6]$.

\subsection{Indentation}

Multiple indents across various section areas were created using the indentation techniques to evaluate the change in Young's modulus and Hardness of the geopolymer pastes due to the acid attack (Figure 12). Analysed section areas included the reference GP and the acid attacked zones having depths of 1-2 mm and 3-4 $\mathrm{mm}$. The results show an increase in both Young's modulus ( $28 \%$ ) and Hardness ( $25 \%$ measured by nanoindents and $12 \%$ by Vickers) with the degree of acid attack. This observation indicates that the sound zone where a significant amount of $\mathrm{Al}$ and $\mathrm{K}$ is leached out (1-4 $\mathrm{mm}$ ) may still possess good mechanical properties, as also observed by visual and manual inspections during sample preparations by sawing, polishing, crushing and grinding. This results are in agreement with literature findings from Koening et al. [9].

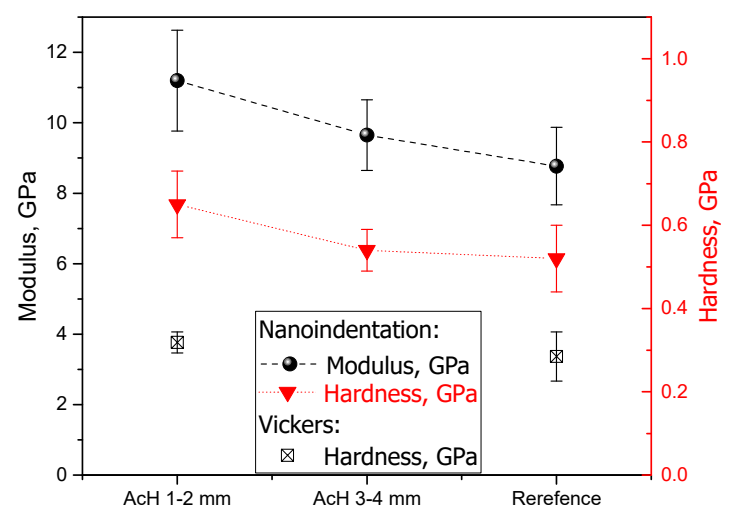

Figure 12. Young's modulus and Hardness results from indentation tests showing the effect of acid attack with degradation depths (1-2 $\mathrm{mm}$ and 3-4 $\mathrm{mm}$ ).

\subsection{X-ray powder diffraction analyses of sliced cross sections}

The acid attack had no major influence on the crystalline phases. No newly built crystalline phases could be detected in all deteriorated layers, which was clearly noticed in its XRD patterns (Figure 13). Traces of muscovite (M), anatase (A) and quartz $(Q z)$ originated from the mineralogical composition of the metakaolin raw material. However, their intensities increased with the degree of acid attack, indicating higher concentrations, in agreement with the leaching of the geopolymer matrix.

The most interesting finding was the shifting of a distinct (GP) amorphous hump towards lower diffraction $(2 \theta)$ angles, in Figure 13 indicated by gray and orange arrows. The shift is the biggest for the surface $(0-1 \mathrm{~mm})$ section depth, while it vanishes at the depth of 2-3 mm, where the pattern shows no significant difference compared to the reference. This result is opposite of a quantitative analysis of geopolymerization [19], which is based on how far an amorphous hump shifts to

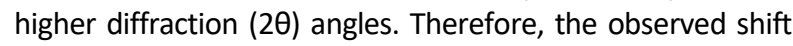
suggest the depolymerization of geopolymer matrix, in agreement with dealumination and hydrolysis process. 


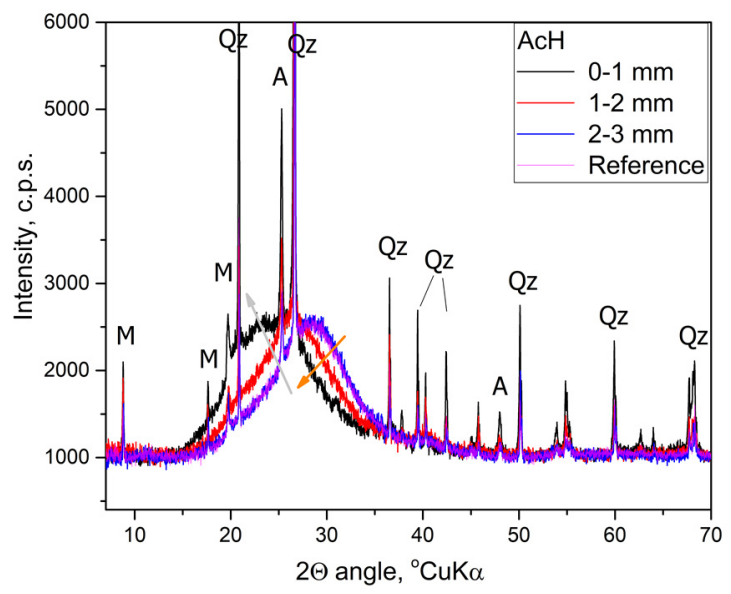

Figure 13. X-ray powder diffraction of sliced cross sections. Notation for identified crystalline phases: M-muscovite, A-Anatase, Qz-quartz.

\section{Conclusion}

The experimental approach for the dissolution rate analysis using the wet-chemistry (ICP) analytics was combined with depth profiles of elemental composition obtained from statistical analysis of SEM-EDS mappings and presented as a powerful tool in investigating the degradation of geopolymers.

The exposure to 0.1 molar acetic acid resulted in about double rates of potassium leaching than for the pure water case. The leaching rates (Figure 4) showed monotonical decrease with time, where the final leaching rates measured after 10 solution replacements were almost two order of magnitudes lower than the initial leaching rates, both in water and acid exposure conditions. This indicated that the degradation process was limited by the diffusion-reaction mechanism that took place within the geopolymer.

The degradation of geopolymer paste in 0.1 molar acetic acid resulted in degradation zone of about $1 \mathrm{~mm}$, characterized by a sharp dissolution of $\mathrm{Al}$, complemented with depletion of potassium $\left(\mathrm{K}^{+}\right)$. The potassium profile exhibited a higher concentration gradient near the exposure surface, observed until the depth of $5.5 \mathrm{~mm}$, considered here as a transition zone, followed by a lower gradient reaching the penetration depth of $9 \mathrm{~mm}$.

The degradation profile after leaching in pure water displayed almost negligible dissolution of $\mathrm{Al}$, but significant dissolution of $\mathrm{Si}$ has reached a degradation depth of about $0.16 \mathrm{~mm}$. This zone exhibited a decrease of Si concentration from about $16.8 \%$ to $10.1 \%$, accompanied with simultaneous depletion of $\mathrm{K}^{+}$, from about $9.55 \%$ to $2.5 \%$.

\section{Acknowledgement}

This research has been supported by TU Darmstadt and the project "PV-WALL" PZS-2019-02-1555 in Research Cooperability Program of the Croatian Science Foundation funded by the European Union from the European Social Fund under the Operational Programme Efficient Human Resources 2014-2020.

\section{CRediT authorship statement}

Neven Ukrainczyk: Conceptualization, Methodology, Validation, Formal analysis, Investigation, Data Curation, Writing, Visualization, Supervision, Project administration, Funding Acquisition.

Oliver Vogt: Resources, Investigation, Writing-review and editing.

\section{References}

[1] Pacheco-Torgal et al.: Handbook of Alkali-activated Cements, Mortars and Concretes. Woodhead pub., Cambridge (2015). https://doi.org/10.1533/9781782422884.1

[2] J. L. Provis, A. Palomo, C. Shi, Advances in understanding alkaliactivated materials. Cem Concr Res (2015) 78: 110-125. https://doi.org/10.1016/i.cemconres.2015.04.013

[3] J. Davidovits, Environmental implications of geopolymers. Materials today Elsevier, 2015.

[4] O. Vogt, N. Ukrainczyk, C. Ballschmiede, E. Koenders, Reactivity and Microstructure of Metakaolin Based Geopolymers: Effect of Fly Ash and Liquid/Solid Contents. Materials (2019) 12: 3485. https://doi.org/10.3390/ma12213485

[5] R.R. Lloyd, J.L. Provis, J.S.J. van Deventer, Pore solution composition and alkali diffusion in inorganic polymer cement, Cem Concr Res (2010) 40: 1386-1392. https://doi.org/10.1016/j.cemconres.2010.04.008

[6] F. Škvára, V. Šmilauer, P. Hlaváček P, L. Kopecký, Z. Cilova, A weak alkali bond in (N,K)-A-S-H gels: evidence from leaching and modeling, Ceramics-Silikaty (2012) 56(4): 374-382.

[7] N. Ukrainczyk, O. Vogt, E.A.B. Koenders, Reactive Transport Numerical Model for Durability of Geopolymer Materials. Advances Chem Eng Sci (2016) 6: 355-363. https://doi.org/10.4236/aces.2016.64036

[8] N. Ukrainczyk, M. Muthu, O. Vogt, E. Koenders, Geopolymer, Calcium Aluminate, and Portland Cement-Based Mortars: Comparing Degradation Using Acetic Acid. Mater (2019) 12: 3115. https://doi.org/10.3390/ma12193115

[9] A. Koenig, A. Herrmann, S. Overmann, F. Dehn, Resistance of alkaliactivated binders to organic acid attack: Assessment of evaluation criteria and damage mechanisms. Constr Build Mater (2017) 151: 405-413. https://doi.org/10.1016/i.conbuildmat.2017.06.117

[10] B. Druga, N. Ukrainczyk, K. Weise, S. Lackner, E.A.B. Koenders, Interaction between wastewater microorganisms and geopolymer or cementitious materials: Biofilm characterization and deterioration characteristics of mortars. Int Biodeterior Biodegradation (2019) 134: 58-67. https://doi.org/10.1016/j.ibiod.2018.08.005

[11] C. Grengg, F. Mittermayr, N. Ukrainczyk, G. Koraimann, S. Kienesberger, M. Dietzel, Advances in concrete materials for sewer systems affected by microbial induced concrete corrosion: A review. Water Research (2018) 134: 341-352. https://doi.org/10.1016/i.watres.2018.01.043

[12] X.X. Gao, P. Michaud, E. Joussein, S. Rossignol, Behavior of metakaolin-based potassium geopolymers in acidic solutions. J Non Cryst Solids (2013) 380: 95-102. https://doi.org/10.1016/j.jnoncrysol.2013.09.002

[13] T. Bakharev, Resistance of geopolymer materials to acid attack. Cem Concr Res (2005) 35(4): 658-670. https://doi.org/10.1016/j.cemconres.2004.06.005

[14] P. Sturm, G. Gluth, C. Jäger, H. Brouwers, H.-C. Kühne, Sulfuric acid resistance of one-part alkali-activated mortars. Cem Concr Res (2018) 109: 54-63. https://doi.org/10.1016/j.cemconres.2018.04.009

[15] C. Grengg, N. Ukrainczyk, G. Koraimann, B. Mueller, M. Dietzel, F. Mittermayr, Long-term in situ performance of geopolymer, calcium aluminate and Portland cement-based materials exposed to microbially induced acid corrosion, Cem Concr Res (2020) 131: 106034. https://doi.org/10.1016/i.cemconres.2020.106034

[16] O. Vogt, C. Ballschmiede, N. Ukrainczyk, E. Koenders, Evaluation of sulfuric acid-induced degradation of potassium silicate activated metakaolin geopolymers by semi-quantitative SEM-EDX analysis. Materials (2020) 13: 4522. https://doi.org/10.3390/ma13204522

[17] C. Grengg, G.J.G. Gluth, F. Mittermayr, N. Ukrainczyk, M. Bertmer, A. Guilherme Buzanich, M. Radtke, A. Leis, M. Dietzel, Deterioration mechanism of alkali-activated materials in sulfuric acid and the influence of $\mathrm{Cu}$ : a micro-to-nano structural, elemental and stable isotopic multi-proxy study. Cem Concr Res, accepted. 
[18] M. Muthu, N. Ukrainczyk, E. Koenders, Effect of graphene oxide dosage on the deterioration properties of cement pastes exposed to an intense nitric acid environment. Constr Build Mater (2020) 121272. https://doi.org/10.1016/j.conbuildmat.2020.121272

[19] R.P. Williams, R.D. Hart, A. Van Riessen, Quantification of the Extent of Reaction of Metakaolin-Based Geopolymers Using X-Ray Diffraction, Scanning Electron Microscopy, and Energy-Dispersive Spectroscopy. J Am Ceram Soc (2011) 94: 2663-2670. https://doi.org/10.1111/j.1551-2916.2011.04410.x 